\title{
The dynamical diquark model: fine structure and isospin
}

\author{
Jesse F. Giron, Richard F. Lebed and Curtis T. Peterson \\ Department of Physics, Arizona State University, \\ Tempe, AZ 85287, U.S.A. \\ E-mail: jfgiron@asu.edu, Richard.Lebed@asu.edu, \\ curtistaylor.peterson@asu.edu
}

ABSTRACT: We incorporate fine-structure corrections into the dynamical diquark model of multiquark exotic hadrons. These improvements include effects due to finite diquark size, spin-spin couplings within the diquarks, and most significantly, isospin-dependent couplings in the form of pionlike exchanges assumed to occur between the light quarks within the diquarks. Using a simplified two-parameter interaction Hamiltonian, we obtain fits in which the isoscalar $J^{\mathrm{PC}}=1^{++}$state - identified as the $X(3872)$ - appears naturally as the lightest exotic (including all states that are predicted by the model but have not yet been observed), while the closed-charm decays of $Z_{c}(3900)$ and $Z_{c}(4020)$ prefer $J / \psi$ and $h_{c}$ modes, respectively, in accord with experiment. We explore implications of this model for the excited tetraquark multiplets and the pentaquarks.

KeYwords: Phenomenological Models, QCD Phenomenology

ARXIV EPRINT: 1907.08546 


\section{Contents}

1 Introduction 1

2 Effects due to finite diquark size 4

$\begin{array}{lll}3 & \text { Isospin interactions between diquarks } & 6\end{array}$

4 Results and analysis $\quad 12$

$\begin{array}{llr}5 & \text { Discussion and conclusions } & 17\end{array}$

\section{Introduction}

The census of heavy-quark ( $c$ - or $b$-containing) exotic hadrons has now reached about 40 candidates, with no indication of a slackening in the pace of their discovery. Equally surprising is that no single theoretical picture has emerged as a global paradigm to describe their structure. Advocates can point to examples among the exotics for which hadronic molecules, hybrids, hadroquarkonium, diquark states, or threshold effects are particularly well suited, while detractors can point to equally compelling counterexamples. The status of both experimental results and theoretical pictures have been reviewed extensively in a number of recent reviews [1-11].

From the theoretical point of view, all of the pictures are based upon sound ideas - phenomena either proven to exist in phenomenology (e.g., atomic nuclei as hadronic molecules) or as well-studied features of quantum field theory [e.g., the $\mathrm{SU}(3)_{\text {color-triplet }}$ diquark attraction; hadronic on-shell threshold-induced singularities in Green's functions from chiral Lagrangians]. However, which particular mechanisms are most important to describe the detailed spectrum and decay modes of the existing exotics remains an unsettled and hotly disputed question. Even if one specific picture eventually emerges as the dominant model, very likely the inclusion of modifications due to the other effects - i.e., full coupled-channel calculations — will be essential in order to obtain a detailed understanding of the exotics.

In this spirit, it is essential to push any given theoretical picture to its limit, examining both its successes and shortcomings as a global model for the exotics. The purpose of the current work is to continue the development of the dynamical diquark picture of exotics [12, 13], which is defined through the color attraction of the channel $\mathbf{3} \otimes \mathbf{3} \rightarrow \overline{\mathbf{3}}$ to form heavy-light diquarks $\delta \equiv(Q q)_{\overline{\mathbf{3}}}$ and their antiparticles $\bar{\delta} \equiv(\bar{Q} \bar{q})_{\mathbf{3}}$ as quasi-bound hadronic subcomponents. The presence of a heavy quark $Q$ means that $\delta$ is more spatially compact than a typical light-quark hadron, while the large energy release available in the production processes of exotics (either through $b$-quark decay [for charmoniumlike states] or collider production) means that the color-nonsinglet $\delta$ and $\bar{\delta}$ can separate a sufficient distance 
to allow the $\delta-\bar{\delta}$ state to temporarily evade color recombination (into, e.g., a hadronic molecule) until the quarks of $\delta$ ultimately combine with the antiquarks of $\bar{\delta}$ in the decay of the state. In other words, ref. [12] argues that when a collection of quarks $Q \bar{Q} q \bar{q}$ forms, the dominant decay mode is indeed two mesons, with the formation of a di-meson hadronic molecule being a rare special case. But if $Q, q$ initially lie closer to each other than to $\bar{Q}, \bar{q}$, then $\delta$ and $\bar{\delta}$ formation is an alternative. If the $\delta-\bar{\delta}$ pair were created with low relative momentum within a typical hadronic volume, then such a state would still exhibit a large overlap with the two-meson wave function. In that case nothing would impede a very rapid decay to this mode, obscuring the fact that a $\delta-\bar{\delta}$ pair had initially formed. But if a large relative momentum occurs in the production process to push the diquarks apart before this recombination can occur, then the two-meson wave-function overlap is suppressed, the decay is delayed, and the appearance of a $\delta-\bar{\delta}$ state becomes perceptible. Put another way, the momentum-space wave function of the two diquarks overlaps more strongly with two-meson states in its small-momentum regime, and with $\delta$ - $\bar{\delta}$ states in its large-momentum regime. Moreover, the triplet-channel attraction need not conclude after just two quarks [14], leading to the proposal of triquarks $\bar{\theta} \equiv\left[\bar{Q}\left(q_{1} q_{2}\right)_{\overline{\mathbf{3}}}\right]_{\mathbf{3}}$ as components of pentaquark states in the combination $\bar{\theta} \delta$ [13].

The dynamical diquark picture has been developed into a full model, including a specific spectroscopy and decay selection rules, in ref. [15]. The key ingredient necessary to characterize states formed from separated $\delta-\bar{\delta}$ or $\bar{\theta}-\delta$ pairs is the introduction of the BornOppenheimer (BO) approximation [16], which distinguishes the heavy, slowly changing $\delta$ and $\bar{\delta}$ (or $\bar{\theta}$ ) from the rapidly changing degrees of freedom in the color flux tube connecting them. The spectrum of flux-tube configurations of nontrivial gluon content has, in turn, been studied on the lattice for decades; for example, these simulations have been used to compute heavy-quarkonium hybrid-meson masses [17].

In ref. [18], the results of lattice simulations obtained by two independent collaborations $[19,20]$ for two separated, color-triplet sources have been input as static-source BO potentials $V(r)$ for Schrödinger equations of $\delta-\bar{\delta}$ and $\bar{\theta}-\delta$ systems. Any observed exotic of known mass and $J^{\mathrm{PC}}$ quantum numbers may then be identified with a state of the same $J^{\mathrm{PC}}$ appearing in one of the multiplets listed in ref. [15] and selected as a reference state, its mass serving as a particular eigenvalue of the Schrödinger equations, which for tetraquarks fixes the diquark mass $m_{\delta}$. But then, with $V(r)$ and $m_{\delta}$ specified, the entire mass spectrum of all tetraquarks is completely determined - at least, ignoring the finestructure mass splittings within the levels of each BO potential. If one chooses the $1^{++}$ $X(3872)$ to fix the (positive-parity) ground-state multiplet $\Sigma_{g}^{+}(1 S)$, then ref. [18] shows that the (negative-parity) first excited levels $\Sigma_{g}^{+}(1 P)$ appear at about $4240 \mathrm{MeV}$, in excellent agreement with $1^{--}$states such as the $Y(4220)$ appearing nearby, and the next (positive-parity) excited levels $\Sigma_{g}^{+}(2 S)$ appear at about $4440 \mathrm{MeV}$, in excellent agreement with the appearance of the $1^{+-}$state $Z_{c}(4430)$. Pentaquarks can then be studied by using the value of $m_{\delta}$ obtained from the tetraquark fit to select a reference pentaquark state to fix $m_{\bar{\theta}}$, and hence, predict the rest of the spectrum.

To go further with this analysis, however, one must consider the aforementioned finestructure corrections. Just as for quarkonium, one can identify multiple types of such cor- 
rections: spin-spin, spin-orbit, tensor, Darwin terms, etc...However, multiquark exotics offer a much richer possible set of interactions, simply due to the greater combinatorics available to their constituent particles. Choosing to work with a diquark model simplifies matters somewhat, by clustering the components into identifiable subunits with good quantum numbers. For example, ref. [21] achieved rather satisfying results in their "TypeII" diquark model by assuming that the dominant spin-spin interactions are solely those between the quarks within each diquark; the mass splitting between the two $1^{+-}$states $Z_{c}^{0}(3900)$ and $Z_{c}^{0}(4020)$ arises quite naturally in this scheme.

One ingredient that, to our knowledge, has not before been included in previous diquark models is isospin dependence in the interaction potential. In the most naive type of tetraquark model, two quarks and two antiquarks are placed in close proximity, and (in the limit $m_{u}=m_{d}$ ) one expects no distinction between tetraquarks differing only in the light-flavor contents $u \bar{u}, u \bar{d}, d \bar{u}$, and $d \bar{d}$. That is, one expects completely degenerate quartets consisting of $I=0$ and $I=1$ multiplets. But the physical exotics appear to form ordinary $I=0$ and $I=1$ multiplets (the experimental absence [22] of a charged partner to the $X(3872)$ is particularly significant in this respect), so a truly predictive model of exotics must contain isospin-dependent effects at some level. Since the $\delta-\bar{\delta}$ pair is connected strongly by color-nonsinglet interactions, one expects the same for the isospin-exchange quanta in this model (Such an assumption however is not strictly necessary for the analysis presented below; the phenomenological consequences of assuming purely color-singlet isospin exchanges between the component quarks of hadrons have been successfully studied for quite some time [23]). In the context of dense QCD, a variant of the Nambu-Goldstone theorem has been demonstrated [24], which means that light pionlike exchange (colored, in this case) can exist in settings other than that of the zero-density environment between color-singlet hadrons. If one posits that the interior of the color flux tube connecting the $\delta$ $\bar{\delta}$ pair is another such environment, then light "pions", possibly partly colored, could exist and propagate across the color flux tube, evading the strong color screening that might impede the propagation of ordinary color-singlet mesons, and providing the essential isospin dependence in these states. We emphasize that such an effect is purely conjectural at this stage, but discuss later how its existence might be established on the lattice.

In the ground-state multiplet $\Sigma_{g}^{+}(1 S)$, the 6 possible states [see eq. (3.5) or (3.6) below] should therefore actually be listed as 6 isosinglets and 6 isotriplets, for a total of 12 mass eigenstates (when $m_{u}=m_{d}$ ). Likewise, one finds 28 mass eigenstates for the first excited $\left[\Sigma_{g}^{+}(1 P)\right]$ multiplet and another 12 for the second excited $\left[\Sigma_{g}^{+}(2 S)\right]$ multiplet. Such large multiplicities have led to the most frequent criticism of diquark models, that they tend to overproduce states compared to experiment.

In this regard, however, several points should be noted: first, new exotic states are still being discovered or resolved - even at relatively low masses — virtually every year, so it is not at all impossible that the final tally in any flavor sector may turn out to be well over 100. Second, some of the predicted states have $J^{\mathrm{PC}}$ quantum numbers that may be difficult to probe with available production channels (e.g., the conventional $\psi_{3}(1 D)$ $\left(3^{--}\right)$charmonium candidate state $X(3842)$ has only been observed for the first time this year [25]). Third, if a state lies only a modest amount above its fall-apart decay threshold, 
then it can be quite wide, and possibly difficult to distinguish from background (e.g., the conventional charmonium $\chi_{c 0}(2 P)$ candidate state $\chi_{c 0}(3860)$ lies only about $130 \mathrm{MeV}$ above the $D \bar{D}$ threshold but has a width of about $200 \mathrm{MeV}$ [26], which made it challenging to resolve until relatively recently).

The second common criticism of such models is that the diquark quasiparticles are not pointlike (estimated radii of a few times $0.1 \mathrm{fm}[12]$ ), and if the full exotic states are not too many times larger, then the $\delta$ and $\bar{\delta}$ wave functions must have considerable spatial overlap. But then, one expects that the stronger $q \bar{q}$ color-singlet attractions should lead to a rearrangement of the quark constituents into a configuration resembling a hadron molecule or hadroquarkonium (see, e.g., [27]). That is to say, for small $\delta-\bar{\delta}$ separations (or small heavy-meson separations in a molecular model), the $Q \bar{Q}$ pair lie in close proximity within the cloud of the $q \bar{q}$ pair, and the nature of the wave function resembles that of hadroquarkonium, with these three pictures distinguished only by the specific color correlations of the quark pairs. In the original dynamical diquark model, such a color reorganization prior to decay is suppressed by the separation of the $\delta \bar{\delta}$ pair. One can also develop models in which this separation is not merely the result of the production process, but is enforced by a potential barrier [28].

In this work we also explore the effect of finite diquark sizes by modeling the Schrödinger equations to transition at a chosen distance $R$ from ones describing the interaction of the $\delta \bar{\delta}$ pair to ones describing just the interaction of the $Q \bar{Q}$ pair. Then the exotic consists primarily of an interacting $Q \bar{Q}$ pair residing in a shell of constant potential provided by the light $q \bar{q}$ pair and glue, which is indeed quite similar to the hadroquarkonium picture. We see below that the calculated spectrum is fairly insensitive to changes of $R$ from zero to physically reasonable values, providing confidence in this aspect of the modeling of $\delta-\bar{\delta}$ states.

This paper is organized as follows. In section 2 we examine the effect of finite diquark size on the exotics spectrum in the manner just described. The introduction of isospindependent interactions between the $\delta-\bar{\delta}$ pair appears in section 3 , and we compute the corresponding expressions for the spectrum of the ground-state $\Sigma_{g}^{+}(1 S)$ multiplet, including both isospin and spin-spin dependence. In section 4 we fit the $X(3872), Z_{c}(3900)$, and $Z_{c}(4020)$ states to the model parameters, and show that natural choices of the unfixed parameters allow all unconfirmed members of the multiplet to lie higher in mass, and indeed respect the pattern of $Z_{c}(3900) / Z_{c}(4020)$ closed-charm decay modes. Finally, in section 5 we indicate the direction of the analogous investigation for excited multiplets, pentaquarks, and the $b \bar{b}$ sector, and summarize our findings.

\section{Effects due to finite diquark size}

The calculations of ref. [18] assume a potential $V(r)$ valid for a $\delta$ - $\bar{\delta}$ pair that can assume any separation $r$. The functional form $V(r)$ is taken from lattice simulations for a heavy (hence static) particle pair transforming as $\mathbf{3}$ and $\overline{\mathbf{3}}$ under $\mathrm{SU}(3)_{\text {color }}$. The specific masses, spin statistics, flavor, and charge quantum numbers of the heavy sources are considered immaterial to the results of these calculations, and so one may use the same potentials 
for heavy $Q \bar{Q}$ states (using the ground-state $\mathrm{BO}$ potential $\Sigma_{g}^{+}$of the color flux tube) or their hybrids $Q \bar{Q} g$ (using the excited BO potentials such as $\Pi_{u}^{+}, \Sigma_{u}^{-}$, etc.), or for $\delta \bar{\delta}$ tetraquark and $\bar{\theta} \delta$ pentaquark states. Of course, quarks are fundamental, presumably pointlike constituents, while diquarks and triquarks have a finite spatial extent. One should not expect that the same potential $V(r)$ as used for interactions between pointlike sources should hold $\delta-\bar{\delta}$ or $\bar{\theta}-\delta$ pairs at arbitrarily small values of $r$, in regions where the wave functions of the quasiparticles strongly overlap.

We present a simple proposal to test the effect of the finite diquark (or triquark) size: since each such quasiparticle in this model contains exactly one heavy quark or antiquark, we suppose for simplicity the existence of a critical separation $R$ between the centers of the $\delta-\bar{\delta}$ or $\bar{\theta}-\delta$ pair, at which point the wave-function overlap between the two is considered significant. Were the quasiparticles hard spheres, then $R$ would equal the sum of their radii. At distances $r<R$, we suppose that the dominant interaction becomes the attraction between the $Q \bar{Q}$ pair, which uses precisely the same $V(r)$ as for $\delta \bar{\delta}$ or $\bar{\theta} \delta$ since it is also

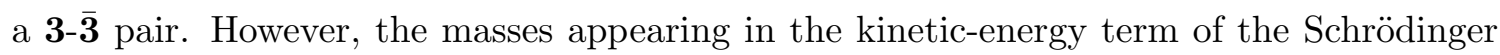
equation are no longer $m_{\delta}$ or $m_{\bar{\theta}}$, but $m_{Q}$. We further suppose that, at reasonably small $R$, the $q \bar{q}$ pair simply provides a constant potential in which the $Q \bar{Q}$ pair interact. Since the state then consists of a $Q \bar{Q}$ pair within a light cloud consisting of the $q \bar{q}$ pair and glue, the physical picture becomes quite similar to that of hadroquarkonium [29].

Explicitly, the Hamiltonian used in the Schrödinger equation assumes the usual form $H=\frac{p^{2}}{2 \mu}+V(r)$, where $p$ is the relative momentum of the constituents, and $V(r)$ is the specific lattice-computed $\mathbf{3}-\overline{\mathbf{3}}$ potential chosen for the calculation holding for all $r$. However,

$$
\frac{1}{\mu}=\left\{\frac{1}{m_{\delta}}+\frac{1}{m_{\bar{\delta}}} \text { for } r>R, \text { and } \frac{1}{m_{Q}}+\frac{1}{m_{\bar{Q}}} \text { for } r<R\right\},
$$

and matching at $r=R$ is accomplished by imposing continuity of the eigenfunction and its first derivative. The values of $m_{\delta}, m_{\bar{\delta}}$ are then adjusted to obtain the physical mass eigenvalue [e.g., $m_{X(3872)}$ ]. One may of course introduce any one of a number of different methods with a variety of refinements to incorporate the finite size of the diquark, but this simple ansatz provides a convenient one-parameter $(R)$ method of testing the limitations of the approach.

At $R=0$, the diquark becomes pointlike. One then recovers the results calculated in ref. [18], specifically the first fits of table 3 (within small numerical tolerances), in which the $\Sigma_{g}^{+}(1 S)$ mass eigenvalue is fixed to that of the $X(3872)$, the diquark mass $m_{\delta}$ entering the Schrödinger equation is obtained as an output, and the charm-quark mass is fixed to a typical value, $m_{c}=1.477 \mathrm{GeV}[30]$. We have computed modifications to the spectrum using the above ansatz and a variety of values of $R$ ranging from $0 \rightarrow 1 \mathrm{fm}$ (corresponding to a classical hard-sphere diquark radius of $0.5 \mathrm{fm}$ ). Sample results are presented in table 1 ; the right-hand columns $(R=0.0 \mathrm{fm})$ reproduce the results of ref. [18], and the left-hand columns are computed at $R=0.7 \mathrm{fm}$. The acronyms refer to the results of lattice simulations by two collaborations, JKM [19, 31] and CPRRW [20].

One immediately notes how little many of the numerical results change. The value of $m_{\delta}$, for example, decreases by a percent or less. The $2 S-1 S$ mass splitting decreases by 


\begin{tabular}{|c|c|c|c|c|c|c|c|c|c|}
\hline \multirow[b]{2}{*}{ BO states } & \multirow[b]{2}{*}{ Potential } & \multicolumn{4}{|c|}{$R=0.7 \mathrm{fm}$} & \multicolumn{4}{|c|}{$R=0.0 \mathrm{fm}$} \\
\hline & & $M$ & $m_{\delta}$ & $\langle 1 / r\rangle^{-1}$ & $\langle r\rangle$ & $M$ & $m_{\delta}$ & $\langle 1 / r\rangle^{-1}$ & $\langle r\rangle$ \\
\hline \multirow[t]{2}{*}{$\Sigma_{g}^{+}(1 S)$} & JKM & 3.8716 & 1.8556 & 0.36925 & 0.32136 & 3.8716 & 1.8750 & 0.27202 & 0.36461 \\
\hline & CPRRW & 3.8717 & 1.8390 & 0.36780 & 0.32538 & 3.8716 & 1.8532 & 0.27521 & 0.36915 \\
\hline \multirow[t]{2}{*}{$\Sigma_{g}^{+}(2 S)$} & JKM & 4.4231 & 1.8556 & 0.49495 & 0.65605 & 4.4435 & 1.8750 & 0.42698 & 0.69081 \\
\hline & CPRRW & 4.4256 & 1.8390 & 0.49385 & 0.66085 & 4.4405 & 1.8532 & 0.43064 & 0.69640 \\
\hline \multirow[t]{2}{*}{$\Sigma_{g}^{+}(1 P)$} & JKM & 4.2067 & 1.8556 & 0.60909 & 0.50400 & 4.2462 & 1.8750 & 0.48962 & 0.56613 \\
\hline & CPRRW & 4.2072 & 1.8390 & 0.60589 & 0.50798 & 4.2429 & 1.8532 & 0.49376 & 0.57067 \\
\hline \multirow[t]{2}{*}{$\Sigma_{g}^{+}(1 D)$} & JKM & 4.4863 & 1.8556 & 0.75652 & 0.67579 & 4.5323 & 1.8750 & 0.66419 & 0.73132 \\
\hline & CPRRW & 4.4881 & 1.8390 & 0.75436 & 0.67993 & 4.5277 & 1.8532 & 0.66931 & 0.73656 \\
\hline
\end{tabular}

Table 1. Mass eigenvalues $M$ (in $\mathrm{GeV}$ ) for hidden-charm dynamical diquark states that are eigenstates (with quantum numbers $n L$ ) of a Schrödinger equation in which $V(r)$ is the ground-state BO potential $\Sigma_{g}^{+}$. The functional form of $V(r)$ is given by lattice simulations JKM [19, 31] or CPRRW [20]. The eigenvalue for the $1 S$ state is fixed to the $X(3872)$ mass, and the diquark mass $m_{\delta}$ (in $\mathrm{GeV}$ ) is the parameter that must be used as input for the Schrödinger equation in order to achieve this constraint. As described in the text, this equation uses $m_{\delta}$ as its mass parameter for $r>R$, and $m_{c}=1.477 \mathrm{GeV}$ for $r<R$ (the separation between $\delta$ and $\bar{\delta}$ centers). Also computed are the corresponding expectation values for the length scales $\langle 1 / r\rangle^{-1}$ and $\langle r\rangle$ (in fm).

only $15-20 \mathrm{MeV}$ in going from $R=0.0 \mathrm{fm}$ to $R=0.7 \mathrm{fm}$, the $1 P-1 S$ splitting decreases by $36-40 \mathrm{MeV}$, and even the $1 D-1 S$ splitting decreases by no more than $46 \mathrm{MeV}$. These changes amount to roughly $3-12 \%$ decreases in the overall size of the splittings, with the largest effect occurring in the $1 P-1 S$ splitting. It is only for $R>0.8 \mathrm{fm}$ that one begins to see the results changing more dramatically, so we take $0.4 \mathrm{fm}$ as an indication of the largest diquark radius one may reasonably use as quasiparticles in these calculations. The length-scale expectation values, on the other hand, change quite drastically with $R$; but since $\langle r\rangle$, for example, is a convolution of the average distance between the $\delta-\bar{\delta}$ pair (for $r>R$ ) with the average distance between the $Q \bar{Q}$ pair (for $r<R$ ), it is not surprising that $\langle r\rangle$ is sensitive to changing the mass parameter in the Schrödinger equation from $m_{\delta}$ to $m_{Q}$.

\section{Isospin interactions between diquarks}

The one-pion exchange potential between two spin- $\frac{1}{2}$ nucleons (with corresponding spin $\boldsymbol{\sigma}$ and isospin $\boldsymbol{\tau}$ operators), separated by a relative position vector $\boldsymbol{r}$, has been known for many decades (arguably, as early as 1938 [32, 33]). In modern notation, it reads:

$$
\begin{array}{r}
V_{\pi}(\boldsymbol{r})=\left(\frac{g_{A}}{\sqrt{2} f_{\pi}}\right)^{2} \boldsymbol{\tau}_{1} \cdot \boldsymbol{\tau}_{2}\left[\frac{m_{\pi}^{2}}{12 \pi} \frac{e^{-m_{\pi} r}}{r}\left(\boldsymbol{\sigma}_{1} \cdot \boldsymbol{\sigma}_{2}+S_{12}\left[1+\frac{3}{m_{\pi} r}+\frac{3}{\left(m_{\pi} r\right)^{2}}\right]\right)\right. \\
\left.-\frac{1}{3} \boldsymbol{\sigma}_{1} \cdot \boldsymbol{\sigma}_{2} \delta^{(3)}(\boldsymbol{r})\right]
\end{array}
$$

where the tensor operator $S_{12}$ is defined by

$$
S_{12} \equiv 3 \boldsymbol{\sigma}_{1} \cdot \boldsymbol{r} \boldsymbol{\sigma}_{2} \cdot \boldsymbol{r} / r^{2}-\boldsymbol{\sigma}_{1} \cdot \boldsymbol{\sigma}_{2} .
$$


In particular, each term depends upon isospin exchange $\left(\boldsymbol{\tau}_{1} \cdot \boldsymbol{\tau}_{2}\right)$, as well as upon spin exchange, between the nucleons. $S_{12}$ is a rank-2 tensor operator in both spin and position space, and therefore by the Wigner-Eckart theorem all states in an $S$ wave connect through $S_{12}$ only to $D$-wave states, which are expected to lie much higher in energy [18]. $P$-wave states, on the other hand, have nonvanishing diagonal $S_{12}$ matrix elements. The contact term $\delta^{(3)}(\boldsymbol{r})$ is included for formal reasons, but the one-pion exchange potential has long been known [34] to require major modifications at separations below about $2 \mathrm{fm}$, so that the $\delta^{(3)}(\boldsymbol{r})$ term should actually be replaced by explicit short-distance effects. In any case, this term still carries the same spin and isospin dependence as the long-distance potential.

Since the $\sim 2 \mathrm{fm}$ range approximately equals the sum of two nucleon radii (as indicated by, e.g., their $\approx 0.86 \mathrm{fm}$ magnetic radii [35]), one may suppose that a major factor in the transition from the one-pion-exchange region to that of heavier-meson or multi-pion exchanges is the appearance of a substantial overlap of nucleon wave functions.

In eq. (3.1), the experimental value of the axial nucleon-pion coupling is $g_{A}=$ $1.2732(23)$, and the pion decay constant (in this normalization) is $f_{\pi}=130.2(1.7) \mathrm{MeV}$ [35]. Using an isospin-averaged pion mass, we find

$$
\left(\frac{g_{A}}{\sqrt{2} f_{\pi}}\right)^{2} \frac{m_{\pi}^{2}}{12 \pi}=4.72 \mathrm{MeV} \cdot \mathrm{fm}
$$

a value to be used below as a comparison with the strength of the isospin exchange between diquarks.

The exchange of pions (and other mesons) between color-singlet hadrons to bind hadronic molecules, both in the form of potential exchanges such in as the $N N \pi$ interaction discussed above, and in calculations employing chiral Lagrangians, has long been one of the primary mechanisms used to study multiquark exotic hadrons [5]. The long range of pion interactions of course stems from its status as the lightest meson, which in turn follows from its role as a Nambu-Goldstone boson of chiral symmetry breaking. The long-distance isospin dependence of the interactions in molecular models follows primarily from the isospin content of exchanged pions.

We now turn to the analogous interaction for diquarks. In the eigenstates of the dynamical diquark model, the $\delta-\bar{\delta}$ pair assume a nonzero separation, and each of $\delta, \bar{\delta}$ contains a light quark that carries isospin $I=\frac{1}{2}$. However, $\delta$ and $\bar{\delta}$ are color nonsinglets, and they are connected by a color flux tube. The question then becomes whether isospin-dependent exchanges can occur in the environment of nonzero color charge. In fact, a related question was addressed some time ago in the context of high-density QCD. As shown in the context of color-flavor locking, the Nambu-Goldstone theorem of chiral-symmetry breaking remains valid even within this environment of high quark density, so that colored analogues of pions have been shown to exist in this case [24]. In this work we propose that a similar effect arises along an extended color flux tube: in this dense, colored environment of extended spatial size, gluons remain the dominant component, but the formation of a partly colored quark condensate subcomponent in this non-vacuum environment is a very real physical possibility. An analogue to pion exchange would then exist between the $\delta-\bar{\delta}$ pair, providing a natural source of isospin dependence in the exotics spectrum of the dynamical diquark 
model. To be clear, we do not take this effect in the current scenario of the $\delta-\bar{\delta}$ interaction in any sense to be proven to exist, but we do consider such an isospin-dependent interaction with pionlike couplings to be a plausible physical phenomenon. Lattice simulations could provide evidence for such an effect: one could, for example, fix in space two heavy diquarks carrying nontrivial isospin quantum numbers, calculate their interaction potential energy, and examine whether any part of it is flavor non-universal.

Assuming then that the color flux tube connecting the $\delta-\bar{\delta}$ pair supports exchanges of a Nambu-Goldstone boson, one expects a potential interaction between the light flavors within the diquarks similar in form to eq. (3.1). In this paper we ignore the $S_{12}$ term, since the fit is confined to the lowest $S$-wave multiplet. However, nothing in principle prevents an analysis of the $P$-wave (or higher) states; only a paucity of confirmed states in this multiplet discourages such a study at this time, and we provide a few relevant comments on a $P$ or higher-wave analysis in section 5. Neglecting $S_{12}$, the remaining terms of eq. (3.1) are proportional to $\boldsymbol{\tau}_{q} \cdot \boldsymbol{\tau}_{\bar{q}} \boldsymbol{\sigma}_{q} \cdot \boldsymbol{\sigma}_{\bar{q}}$. In the current model, we simply label the coefficient of this operator as $V_{0}$.

One may take the phenomenology a step further in order to compare to ordinary onepion exchange. As discussed above, we strike the contact term $\delta^{(3)}(\boldsymbol{r})$ from the exchange potential, since at short distances the diquark wave functions must overlap, necessarily leading to a more complicated interaction. Next, since the calculations of ref. [18] show how to compute any expectation value without the need of calculating explicit wave functions, one may obtain an explicit expectation value for the Yukawa part of the potential, $\left\langle e^{-m_{\pi} r} / r\right\rangle$, and from this result, extract a coefficient called $\tilde{V}_{0}$ that may be compared with the combination in eq. (3.3). Explicitly, we write the full isospin-dependent potential $V_{I}(r)$ as

$$
\begin{aligned}
V_{I}(r) & =\tilde{V}_{0} \times \frac{e^{-m_{\pi} r}}{r} \times \boldsymbol{\tau}_{q} \cdot \boldsymbol{\tau}_{\bar{q}} \boldsymbol{\sigma}_{q} \cdot \boldsymbol{\sigma}_{\bar{q}}, \\
V_{0} & \equiv \tilde{V}_{0}\left\langle\frac{e^{-m_{\pi} r}}{r}\right\rangle .
\end{aligned}
$$

It bears mentioning that the Yukawa potential expectation value decreases for excited states, and so while one may suppose that $\tilde{V}_{0}$ should be approximately the same constant for all multiplets, the particular value of $V_{0}$ obtained below [eq. (4.7)] holds only for the $\Sigma_{g}^{+}(1 S)$ multiplet. An analogous effect explains why fine-structure splittings in ordinary quarkonium decrease for higher multiplets. A direct comparison with eq. (3.3) is also difficult because the relation between the observed (vacuum) pion mass $m_{\pi}$ and the mass parameter for the corresponding in-medium exchange quantum (the "partly colored pion") along the color flux tube is unknown, not to mention the size of its coupling to the diquark (the analogue to $g_{A} / f_{\pi}$ ). Purely for sake of comparison, we take the mass of the "partly colored pion" in eq. (3.4) to equal $m_{\pi}$, even the true mechanism of isospin exchange might be quite different. One may expect on the basis of ordinary hadronic phenomenology that the proper mass scale of the colored exchange is rather larger (at least several hundred $\mathrm{MeV}$ ), but using such a value does not radically alter our numerical results. 
The states in the ground-state multiplet $\Sigma_{g}^{+}(1 S)$, prior to introducing isospin, are defined as [21]

$$
\begin{array}{ll}
J^{\mathrm{PC}}=0^{++}: & X_{0} \equiv\left|0_{\delta}, 0_{\bar{\delta}}\right\rangle_{0}, \quad X_{0}^{\prime} \equiv\left|1_{\delta}, 1_{\bar{\delta}}\right\rangle_{0}, \\
J^{\mathrm{PC}}=1^{++}: & X_{1} \equiv \frac{1}{\sqrt{2}}\left(\left|1_{\delta}, 0_{\bar{\delta}}\right\rangle_{1}+\left|0_{\delta}, 1_{\bar{\delta}}\right\rangle_{1}\right), \\
J^{\mathrm{PC}}=1^{+-}: & Z \equiv \frac{1}{\sqrt{2}}\left(\left|1_{\delta}, 0_{\bar{\delta}}\right\rangle_{1}-\left|0_{\delta}, 1_{\bar{\delta}}\right\rangle_{1}\right) \\
Z^{\prime} \equiv\left|1_{\delta}, 1_{\bar{\delta}}\right\rangle_{1}, \\
J^{\mathrm{PC}}=2^{++}: \quad X_{2} \equiv\left|1_{\delta}, 1_{\bar{\delta}}\right\rangle_{2},
\end{array}
$$

where the number preceding each $\delta(\bar{\delta})$ subscript is the diquark (antidiquark) spin $\left(s_{\delta}\right.$ and $s_{\bar{\delta}}$, respectively), while the outer subscript on each ket is the total quark spin $J$. In terms of the basis of good $q \bar{q}$ and $Q \bar{Q}$ spin quantum numbers $\left(s_{q \bar{q}}\right.$ and $s_{Q \bar{Q}}$, respectively), the corresponding eigenstates are

$$
\begin{aligned}
\tilde{X}_{0} & \equiv\left|0_{q \bar{q}}, 0_{Q \bar{Q}}\right\rangle_{0}=+\frac{1}{2} X_{0}+\frac{\sqrt{3}}{2} X_{0}^{\prime}, \\
\tilde{X}_{0}^{\prime} & \equiv\left|1_{q \bar{q}}, 1_{Q \bar{Q}}\right\rangle_{0}=+\frac{\sqrt{3}}{2} X_{0}-\frac{1}{2} X_{0}^{\prime}, \\
\tilde{Z} & \equiv\left|1_{q \bar{q}}, 0_{Q \bar{Q}}\right\rangle_{1}=\frac{1}{\sqrt{2}}\left(Z^{\prime}+Z\right), \\
\tilde{Z}^{\prime} & \equiv\left|0_{q \bar{q}}, 1_{Q \bar{Q}}\right\rangle_{1}=\frac{1}{\sqrt{2}}\left(Z^{\prime}-Z\right) .
\end{aligned}
$$

Expressing the basis change between eqs. (3.5) and (3.6) in terms of rotation matrices, one finds

$$
\begin{aligned}
& J^{\mathrm{PC}}=0^{++}: \quad\left(\begin{array}{c}
X_{0} \\
X_{0}^{\prime}
\end{array}\right)=\left(\begin{array}{rr}
\cos \frac{\pi}{3} & \sin \frac{\pi}{3} \\
\sin \frac{\pi}{3} & -\cos \frac{\pi}{3}
\end{array}\right)\left(\begin{array}{c}
\tilde{X}_{0} \\
\tilde{X}_{0}^{\prime}
\end{array}\right), \\
& J^{\mathrm{PC}}=1^{++}: \quad X_{1}=\left|1_{q \bar{q}}, 1_{Q \bar{Q}}\right\rangle_{1}, \\
& J^{\mathrm{PC}}=1^{+-}: \quad\left(\begin{array}{c}
Z \\
Z^{\prime}
\end{array}\right)=\left(\begin{array}{rr}
\cos \frac{\pi}{4} & -\sin \frac{\pi}{4} \\
\sin \frac{\pi}{4} & \cos \frac{\pi}{4}
\end{array}\right)\left(\begin{array}{c}
\tilde{Z} \\
\tilde{Z}^{\prime}
\end{array}\right), \\
& J^{\mathrm{PC}}=2^{++}: \quad X_{2}=\left|1_{q \bar{q}}, 1_{Q \bar{Q}}\right\rangle_{2} \text {, }
\end{aligned}
$$

where outer subscripts again indicate total quark spin $J$.

This nomenclature (adapted from ref. [21]) applies to both $I=0$ and $I=1$ states. However, its use may cause confusion because the label $Z$ is usually understood to mean only $I=1$ states, whereas we use $Z$ to mean $1^{+-}$states exclusively. The naming scheme adopted by the Particle Data Group [35] labels the $I=0 J^{++}$states as $\chi_{J}$ and the $I=0$ $1^{+-}$state as $h$, exactly as for conventional quarkonium, while the label $Z$ is reserved for $I=11^{+-}$states, and the yet-unobserved $I=1 J^{++}$states are called $W_{J}$. 
The mass eigenstates formed from the states of degenerate $J^{\mathrm{PC}}$ in eq. (3.5) are defined as

$$
\begin{aligned}
\left(\begin{array}{c}
\bar{X}_{0} \\
\bar{X}_{0}^{\prime}
\end{array}\right) & =\left(\begin{array}{rr}
\cos \theta_{X} & \sin \theta_{X} \\
-\sin \theta_{X} & \cos \theta_{X}
\end{array}\right)\left(\begin{array}{c}
X_{0} \\
X_{0}^{\prime}
\end{array}\right), \\
\left(\begin{array}{c}
\bar{Z} \\
\bar{Z}^{\prime}
\end{array}\right) & =\left(\begin{array}{rr}
\cos \theta_{Z} & \sin \theta_{Z} \\
-\sin \theta_{Z} & \cos \theta_{Z}
\end{array}\right)\left(\begin{array}{c}
Z \\
Z^{\prime}
\end{array}\right) .
\end{aligned}
$$

While it is not logically necessary to require the mixing angles $\theta_{X}$ and $\theta_{Z}$ for these systems to assume the same values in both the $I=0$ and $I=1$ channels, to do so is a reasonable minimal ansatz. As shown below, this ansatz does not conflict with current experimental findings. In particular, each occurrence of the label $Z$ below is understood to apply equally well to the $I=0$ or $I=1$ state, unless a particular $I$ eigenvalue is explicitly appended.

The full model Hamiltonian reads

$$
H=M_{0}+2 \kappa_{q Q}\left(\mathbf{s}_{q} \cdot \mathbf{s}_{Q}+\mathbf{s}_{\bar{q}} \cdot \mathbf{s}_{\bar{Q}}\right)+V_{0} \boldsymbol{\tau}_{q} \cdot \boldsymbol{\tau}_{\bar{q}} \boldsymbol{\sigma}_{q} \cdot \boldsymbol{\sigma}_{\bar{q}}
$$

where $M_{0}$ is the common multiplet mass, computed in ref. [18] using spin- and isospin-blind Schrödinger equations that depend only upon the diquark (or also, in the pentaquark case, triquark) mass and a central potential computed on the lattice from pure-glue configurations. The second term of eq. (3.9) represents the primary interaction of the "Type-II" diquark model [21], with the parameter $\kappa_{q Q}$ representing the strength of the spin-spin couplings within diquarks ( $q$ only to $Q, \bar{q}$ only to $\bar{Q}$ ). Note particularly the assumption that the dominant isospin-dependent potential in eq. (3.9) depends only upon the light-quark spins, rather than the diquark spins; were the diquarks truly pointlike, then the $q(\bar{q})$ would still carry all the isospin of $\delta(\bar{\delta})$, but the $V_{0}$ interaction would be replaced with

$$
\Delta H=V_{1} \boldsymbol{\tau}_{q} \cdot \boldsymbol{\tau}_{\bar{q}} \boldsymbol{\sigma}_{\delta} \cdot \boldsymbol{\sigma}_{\bar{\delta}} .
$$

The particular form of the Hamiltonian eq. (3.9) [or (3.10)] deserves further comment. The original spin-dependent diquark model for heavy-quark exotics (called "Type I" [36]), which emerged when only a few such exotics were known, allows for couplings between all four quarks. As exotics data improved with time, it became apparent that the "Type-I" model was unsuited to describing the full observed spectrum. The ansatz of the "TypeII" model [21] takes the dominant spin couplings to be just those within each diquark (since the diquarks are believed to be more compact than the full hadron), and the model provides a satisfactory understanding of the masses of the states $X(3872), Z_{c}(3900)$, and $Z_{c}(4020)$ - but not, by construction, their isospins. The "Type-II" ansatz is simplest to justify if one appeals to the kinematically induced separation of the $\delta-\bar{\delta}$ pair in the dynamical diquark picture [12], but one could just as easily suppose the existence of a potential barrier separating the $\delta-\bar{\delta}$ pair [28]. A comparison of the spectra of the "Type-II" and molecular models forms the basis of a dedicated study in ref. [37]. The $V_{0}$ term of eq. (3.9) or $V_{1}$ term of eq. (3.10) introduces isospin dependence into the model, and under the assumption of chiral-type couplings, the isospin exchange is linked to additional spin 
dependence. Certainly one could also incorporate, for example, isospin-dependent, spinindependent Hamiltonian operators, but for this initial study the $V_{0}$ and $V_{1}$ operators are designed to assume the most familiar form from chiral dynamics.

The matrix elements of the symmetry-breaking operators in eq. (3.9) are computed easily using standard square-completion tricks. The second term evaluates to

$$
\kappa_{q Q}\left[s_{\delta}\left(s_{\delta}+1\right)+s_{\bar{\delta}}\left(s_{\bar{\delta}}+1\right)-3\right],
$$

which is trivially computed for states expressed in the diquark basis of eq. (3.5), for which the operator is diagonal. The third term of eq. (3.9) evaluates to

$$
V_{0}[2 I(I+1)-3]\left[2 s_{q \bar{q}}\left(s_{q \bar{q}}+1\right)-3\right],
$$

which is trivially computed for states expressed in the total light-quark spin $\left(s_{q \bar{q}}\right)$ basis of eq. (3.6). In the alternative form of eq. (3.10), one obtains instead the contribution

$$
2 V_{1}[2 I(I+1)-3]\left[J(J+1)-s_{\delta}\left(s_{\delta}+1\right)-s_{\bar{\delta}}\left(s_{\bar{\delta}}+1\right)\right],
$$

which again is easily computed in the diquark-spin basis of eq. (3.5).

Using the mass eigenstates defined in eq. (3.8) and the Hamiltonian of eq. (3.9), one immediately computes the masses for the 12 physical states in the $\Sigma_{g}^{+}(1 S)$ multiplet:

$$
\begin{aligned}
& M_{\bar{X}_{0}}^{I=0}=M_{0}-\kappa_{q Q}\left[1+2 \cos \left(2 \theta_{X}\right)\right]+3 V_{0}\left[1-2 \cos \left(2 \theta_{X}+\frac{\pi}{3}\right)\right], \\
& M_{\bar{X}_{0}^{\prime}}^{I=0}=M_{0}-\kappa_{q Q}\left[1-2 \cos \left(2 \theta_{X}\right)\right]+3 V_{0}\left[1+2 \cos \left(2 \theta_{X}+\frac{\pi}{3}\right)\right], \\
& M_{\bar{X}_{0}}^{I=1}=M_{0}-\kappa_{q Q}\left[1+2 \cos \left(2 \theta_{X}\right)\right]-V_{0}\left[1-2 \cos \left(2 \theta_{X}+\frac{\pi}{3}\right)\right], \\
& M_{\bar{X}_{0}^{\prime}}^{I=1}=M_{0}-\kappa_{q Q}\left[1-2 \cos \left(2 \theta_{X}\right)\right]-V_{0}\left[1+2 \cos \left(2 \theta_{X}+\frac{\pi}{3}\right)\right], \\
& M_{X_{1}}^{I=0}=M_{0}-\kappa_{q Q}-3 V_{0}, \\
& M_{X_{1}}^{I=1}=M_{0}-\kappa_{q Q}+V_{0}, \\
& M_{X_{2}}^{I=0}=M_{0}+\kappa_{q Q}-3 V_{0}, \\
& M_{X_{2}}^{I=1}=M_{0}+\kappa_{q Q}+V_{0}, \\
& M_{\bar{Z}}^{I=0}=M_{0}-\kappa_{q Q} \cos \left(2 \theta_{Z}\right)+3 V_{0}\left[1-2 \sin \left(2 \theta_{Z}\right)\right], \\
& M_{\overline{Z^{\prime}}}^{I=0}=M_{0}+\kappa_{q Q} \cos \left(2 \theta_{Z}\right)+3 V_{0}\left[1+2 \sin \left(2 \theta_{Z}\right)\right], \\
& M_{\bar{Z}}^{I=1}=M_{0}-\kappa_{q Q} \cos \left(2 \theta_{Z}\right)-V_{0}\left[1-2 \sin \left(2 \theta_{Z}\right)\right], \\
& M_{\overline{Z^{\prime}}}^{I=1}=M_{0}+\kappa_{q Q} \cos \left(2 \theta_{Z}\right)-V_{0}\left[1+2 \sin \left(2 \theta_{Z}\right)\right] .
\end{aligned}
$$

These 12 masses depend upon a common multiplet mass $M_{0}$, two Hamiltonian parameters $\left(\kappa_{q Q}\right.$ and $\left.V_{0}\right)$, and the mixing angles $\theta_{X, Z}$. At this point, eqs. (3.14) are equally valid 
for $c \bar{c}$ and $b \bar{b}$ tetraquarks, as well as $B_{c}$ tetraquarks if one includes distinct $\kappa_{q c}$ and $\kappa_{q b}$ couplings. Note that the primed and unprimed states interchange under a trivial shift of the mixing angles: $\bar{X}_{0} \leftrightarrow \bar{X}_{0}^{\prime}$ when $\theta_{X} \rightarrow \theta_{X}+\frac{\pi}{2}$, and similarly $\bar{Z} \leftrightarrow \bar{Z}^{\prime}$ when $\theta_{Z} \rightarrow \theta_{Z}+\frac{\pi}{2}$. Therefore, the unprimed and primed states are equally valid for purposes of parametric fitting to the mass spectrum. However, these states remain inequivalent in terms of their content according to $s_{q \bar{q}}$ and $s_{Q \bar{Q}}$ eigenvalues, a distinction that can be probed through their decay modes.

If eq. (3.10) is used instead, then the $V_{0}$ terms of eqs. (3.14) are replaced by the $V_{1}$ terms

$$
\begin{aligned}
& \Delta M_{\bar{X}_{0}}^{I=0}=+12 V_{1}\left[1-\cos \left(2 \theta_{X}\right)\right], \\
& \Delta M_{\bar{X}_{0}^{\prime}}^{I=0}=+12 V_{1}\left[1+\cos \left(2 \theta_{X}\right)\right], \\
& \Delta M_{\bar{X}_{0}}^{I=1}=-4 V_{1}\left[1-2 \cos \left(2 \theta_{X}\right)\right], \\
& \Delta M_{\bar{X}_{0}^{\prime}=1}^{I=1}=-4 V_{1}\left[1+2 \cos \left(2 \theta_{X}\right)\right], \\
& \Delta M_{X_{1}}^{I=0}=+0 \\
& \Delta M_{X_{1}}^{I=1}=+0 \\
& \Delta M_{X_{2}}^{I=0}=-12 V_{1}, \\
& \Delta M_{X_{2}}^{I=1}=+4 V_{1} \\
& \Delta M_{\bar{Z}}^{I=0}=+6 V_{1}\left[1-\cos \left(2 \theta_{Z}\right)\right], \\
& \Delta M_{\bar{Z}^{\prime}}^{I=0}=+6 V_{1}\left[1+\cos \left(2 \theta_{Z}\right)\right], \\
& \Delta M_{\bar{Z}}^{I=1}=-2 V_{1}\left[1-\cos \left(2 \theta_{Z}\right)\right], \\
& \Delta M_{\bar{Z}^{\prime}}^{I=1}=-2 V_{1}\left[1+\cos \left(2 \theta_{Z}\right)\right] .
\end{aligned}
$$

The invariance of $\bar{X}_{0} \leftrightarrow \bar{X}_{0}^{\prime}$ under $\theta_{X} \rightarrow \theta_{X}+\frac{\pi}{2}$, and $\bar{Z} \leftrightarrow \bar{Z}^{\prime}$ under $\theta_{Z} \rightarrow \theta_{Z}+\frac{\pi}{2}$ also holds in this case. However, the most conspicuous feature of eqs. (3.15) is the degeneracy of $X_{1}^{I=0}$ and $X_{1}^{I=1}$. As these states represent the $X(3872)$ candidate and its yet-unseen charged partner [22], one finds that a model in which the diquarks exchange isospin only in their pointlike form as in eq. (3.10) runs afoul of known phenomenology. That these two $V_{1}$ contributions are not just equal but indeed zero follows immediately from eq. (3.13) and the fact [eq. (3.5)] that the $X_{1}$ states $(J=1)$ contain only components in which $s_{\delta}=0$ and $s_{\bar{\delta}}=1$, or vice versa. We therefore analyze as our minimal model the Hamiltonian given by eq. (3.9), which leads to the spectrum given by eq. (3.14). In addition, if one neglects all isospin-independent couplings $\left(\kappa_{q Q}=0\right)$, then eq. (3.14) shows that the isoscalar, spin-2 state $X_{2}^{I=0}$ would be degenerate with the $X(3872)$, again in opposition to known phenomenology.

\section{Results and analysis}

We now test whether this model can accommodate what is known about the ground-state $\left[\Sigma_{g}^{+}(1 S)\right]$ hidden-charm exotics, the $J^{\mathrm{PC}}=1^{++} X(3872)$, and the $1^{+-}$states $Z_{c}(3900)$ and $Z_{c}(4020)$ (the $C$ parity eigenvalue referring to the neutral states). The Particle Data 
Group [35] averages for their masses are

$$
\begin{aligned}
& m_{X(3872)}=3871.69 \pm 0.17 \mathrm{MeV}, \\
& m_{Z_{c}(3900)}=3887.2 \pm 2.3 \mathrm{MeV}, \\
& m_{Z_{c}(4020)}=4024.1 \pm 1.9 \mathrm{MeV} .
\end{aligned}
$$

The $Z_{c}$ states have been observed in both charged and neutral variants, which decay in their closed-charm modes to $\pi^{ \pm}$and $\pi^{0}$, respectively, meaning that they have $I=1$ [and hence $\left.G=C(-1)^{I}=+\right]$. The dominant decays of these states have open charm: $Z_{c}(3900) \rightarrow$ $\bar{D}^{*} D$ and $Z_{c}(4020) \rightarrow \bar{D}^{*} D^{*}[35]$, making an analysis based solely upon isospin not as incisive. On the other hand, we noted above that no charged partner of the $X(3872)$ has been observed despite a dedicated search [22], which suggests $I=0$. However, the $X(3872)$ is widely believed to be unique among all known hadrons in possessing a valence quark content (more $c \bar{c} u \bar{u}$ than $c \bar{c} d \bar{d}$ ) not corresponding to just one $I$ eigenvalue. Its mass is almost precisely equal that of $\bar{D}^{* 0} D^{0}$ (in fact, its dominant decay mode is $\bar{D}^{* 0} D^{0}[35]$ ) but about $8 \mathrm{MeV}$ below that of $\bar{D}^{*+} D^{-}$, while the idealized $I=0$ and $I=1$ combinations of these states are equal admixtures. Likewise, $X(3872)$ has been observed to decay to both the $G=-$ (hence $I=1$ ) final state $\pi^{+} \pi^{-} J / \psi$ and the $G=+$ final states $\omega J / \psi$ $(I=0)$ and (very recently [38]) $\pi^{0} \chi_{c 0}(G=-, I=1)$. The mere facts that $m_{u}<m_{d}$ (and $\left.q_{u} \neq q_{d}\right)$ and that $X(3872)$ lies in the close proximity to the threshold for one particular charge combination appear to be responsible for these fascinating results. In the current model, however, we take $m_{u}=m_{d}$, ignore electromagnetic effects, and treat $X(3872)$ as the unique $I=01^{++}$state in $\Sigma_{g}^{+}(1 S), X_{1}^{I=0}$. Indeed, the same analysis below holds even if the $X(3872)$ is an exact $I=0$ state (an equal admixture of $c \bar{c} u \bar{u}$ and $c \bar{c} d \bar{d}$ ), and its isospin-violating decays are purely the result of the kinematical blocking of the $d \bar{d}$ channel.

Without performing a detailed accounting of every significant source of fine-structure splitting expected to appear in these states, a precise estimate of the numerical uncertainties on our mass predictions is impossible. Nevertheless, if the model is to have any validity, it must incorporate basic phenomenological facts such as the $\sim 20 \mathrm{MeV}$ mass difference $m_{Z_{c}(3900)}-m_{X(3872)}$. One may therefore take $20 \mathrm{MeV}$ as a reasonable upper limit for mass uncertainties in this model.

Using the values in eqs. (4.1) (with uncertainties suppressed) in eqs. (3.14), one obtains

$$
\begin{aligned}
\frac{1}{2}\left(m_{Z_{c}(4020)}+m_{Z_{c}(3900)}\right) & =M_{0}-V_{0}=3955.65 \mathrm{MeV}, \\
\frac{1}{2}\left(m_{Z_{c}(4020)}+m_{Z_{c}(3900)}\right)-m_{X(3872)} & =\kappa_{q c}+2 V_{0}=83.96 \mathrm{MeV}, \\
\frac{1}{2}\left(m_{Z_{c}(4020)}-m_{Z_{c}(3900)}\right) & =\left|\kappa_{q c} \cos 2 \theta_{Z}-2 V_{0} \sin 2 \theta_{Z}\right|=68.45 \mathrm{MeV} .
\end{aligned}
$$

The absolute value in the third expression reflects the fact, noted above, that $Z_{c}(3900)$ and $Z_{c}(4020)$ may be identified with $\bar{Z}_{I=1}$ or $\bar{Z}_{I=1}^{\prime}$ in either order, under the replacement $\theta_{Z} \rightarrow \theta_{Z}+\frac{\pi}{2}$. According to eqs. (3.6)-(3.8), this substitution exchanges the relative amounts of the $s_{Q \bar{Q}}=0$ and $s_{Q \bar{Q}}=1$ components in the mass eigenstates. In particular, $\theta_{Z}=\frac{\pi}{4}$ takes $\bar{Z}$ to the pure $s_{Q \bar{Q}}=0$ eigenstate $\tilde{Z}$ and takes $\bar{Z}^{\prime}$ to the pure $s_{Q \bar{Q}}=1$ eigenstate 
$\tilde{Z}^{\prime}$, while $\theta_{Z}=\frac{3 \pi}{4}$ takes $\bar{Z} \rightarrow \tilde{Z}^{\prime}$ and $\bar{Z}^{\prime} \rightarrow \tilde{Z}$. Since $Z_{c}(3900)$ has been observed to decay to $\pi J / \psi$ and not $\pi h_{c}$, while the reverse is true for $Z_{c}(4020)$ [35], identifying $\bar{Z}_{I=1}$ with $Z_{c}(3900)$, and $\bar{Z}_{I=1}^{\prime}$ with $Z_{c}(4020)$, is best achieved through values $\theta_{Z} \approx \frac{3 \pi}{4}$. Even so, we do not impose this constraint on the fit, focusing initially only upon the mass spectrum.

First, even without information on $\theta_{Z}$, we predict

$$
M_{X_{2}}^{I=1}=m_{Z_{c}(4020)}+m_{Z_{c}(3900)}-m_{X(3872)}=4039.61 \mathrm{MeV} .
$$

Should a charged, $J^{\mathrm{PC}}=2^{++}$exotic state fail to occur in the vicinity of $4040 \mathrm{MeV}$, then the validity of this simplified model must be reassessed. Note that the $X_{2}^{I=1}$ has $G=-1$ and, according to eq. (3.7), preferentially decays to $J / \psi$, which also carries $G=-1$. This state would therefore most easily be seen in the channel $\pi \pi J / \psi$.

Imposing the constraints of eqs. (4.2) on the last 8 mass expressions in eq. (3.14) leaves the remaining four non-scalar states, $M_{X_{1}}^{I=1}, M_{X_{2}}^{I=0}, M_{\bar{Z}}^{I=0}$, and $M_{\bar{Z}^{\prime}}^{I=0}$, as functions of the single parameter $\theta_{Z}$. Taking $\theta_{Z} \rightarrow \theta_{Z}+\pi$ simply changes the normalization sign of both $\bar{Z}$ and $\bar{Z}^{\prime}$, so one may consider only the range $\theta_{Z} \in[0, \pi]$. The most important constraint from a phenomenological perspective is that the "charged partner" to the $X(3872), X_{1}^{I=1}$, must be substantially heavier, at least $20 \mathrm{MeV}$ [22], than the $X(3872)$. From eqs. (3.14), one notes that this constraint simply reads $V_{0}>5 \mathrm{MeV}$. While $V_{0}$ is not yet fixed at this stage of the fit, one notes that

$$
\frac{1}{2}\left(M_{X_{1}}^{I=1}+M_{X_{2}}^{I=0}\right)=M_{0}-V_{0}=3955.65 \mathrm{MeV},
$$

meaning that allowing $X_{1}^{I=1}$ to be excessively heavy forces the spin- 2 isoscalar $X_{2}^{I=0}$ to be so light that it would already have been observed. $\theta_{Z}$ can only be allowed in certain numerical ranges to avoid this problem, but fortunately, these ranges are substantial: the lighter of $\left\{X_{1}^{I=1}, X_{2}^{I=0}\right\}$ exceeds the $X(3872)$ mass for $\theta_{Z} / \pi \in[0,0.10],[0.65,0.85]$, and [0.90, 1.00]. $X_{1}^{I=1}$ is the heavier of these two states in the middle interval and the lighter in the other two intervals. Within these ranges, $M_{X_{1}}^{I=1}-m_{X(3872)}>20 \mathrm{MeV}$ for the restricted ranges $\theta_{Z} / \pi \in[0,0.04],[0.65,0.85]$, and $[0.91,1.00]$. The masses of the two isoscalar partners $\left\{\bar{Z}_{I=0}, \bar{Z}_{I=0}^{\prime}\right\}$ to the $Z_{c}(3900)$ and $Z_{c}(4020)$ exceed $m_{X(3872)}$ over these full ranges, and the lighter of the two exceeds $m_{Z_{c}(3900)}$ over the restricted ranges except for the small interval $\theta_{Z} / \pi \in[0.71,0.75]$, where even there it is never more than about $2 \mathrm{MeV}$ below $m_{Z_{c}(3900)}$; indeed, precisely at the ideal mixing angle $\theta_{Z}=\frac{3 \pi}{4}$, eqs. (3.14) show that the isoscalar $\bar{Z}_{I=0}^{\prime}$ becomes degenerate with $\bar{Z}_{I=1}=Z_{c}(3900)$. One finds, therefore, that rather large ranges of $\theta_{Z}$ appear to all satisfy spectroscopic constraints.

The possibility of an isoscalar $1^{+-}$state quasi-degenerate with the $Z_{c}(3900)$ is interesting in light of phenomenological mystery: $m_{Z_{c}(3900)}$ as determined via its $\pi J / \psi$ decay channel (pure $I=1$ ) tends to lie several $\mathrm{MeV}$ above its value as determined through $\left(D \bar{D}^{*}\right)^{0}$ (a mixture of $\left.I=0,1\right)$ [35]. If the latter resonance turns out to be a mixture of $\bar{Z}_{I=0}^{\prime}$ and $\bar{Z}_{I=1}$, then a shifted mass - an average of the two mass eigenvalues - might be expected. In addition, if $\bar{Z}_{I=1}$ is nearly ideally mixed to decay to $s_{c \bar{c}}=1$ charmonium, then $\bar{Z}_{I=0}^{\prime}$ is nearly ideally mixed to decay to $s_{c \bar{c}}=0$ charmonium, meaning that one would have a mixture of both components in this scenario. However, since the $Z_{c}(3900)^{ \pm}$ 
mass measured through the channel $\left(D \bar{D}^{*}\right)^{ \pm}$(a pure $I=1$ combination) is also low, this resolution is not entirely satisfactory. Nevertheless, such mixing should be kept in mind as a possibility, should the $Z_{c}(3900)$ mass discrepancy persist.

Turning to the decay properties, we have already noted the preferential coupling of $Z_{c}(3900)$ to $J / \psi$ and $Z_{c}$ (4020) to $h_{c}$. As easily seen from combining eqs. (3.6)-(3.8), the $s_{c \bar{c}}=1$ content of $\bar{Z}_{I=1}=Z_{c}(3900)$ is given by

$$
P_{s_{c \bar{c}}=1}\left[Z_{c}(3900)\right]=\sin ^{2}\left(\theta_{Z}-\frac{\pi}{4}\right) .
$$

In the restricted allowed ranges for $\theta_{Z} / \pi$ listed above, we find $P_{s_{c \bar{c}}=1}\left[Z_{c}(3900)\right] \in[0.36,0.50]$, $[0.91,1.00]$, and $[0.50,0.76]$, respectively. In light of the preference for $Z_{c}(3900) \rightarrow J / \psi$ and $Z_{c}(4020) \rightarrow h_{c}$, the second region, $\theta_{Z} / \pi \in[0.65,0.85]$, appears to be favored. Note a very recent result [39], the observation of $Z_{c}(3900)^{ \pm} \rightarrow \rho^{ \pm} \eta_{c}$, indicating that $Z_{c}(3900)$ is not a perfect $s_{c \bar{c}}=1$ state.

We now consider the scalar sector. Recalling that $\bar{X}_{0} \leftrightarrow \bar{X}_{0}^{\prime}$ when $\theta_{X} \rightarrow \theta_{X}+\frac{\pi}{2}$ for both the $I=0,1$ channels, one need consider only the range $\theta_{X} / \pi \in\left[0, \frac{1}{2}\right]$. One then finds, over the preferred range $\theta_{Z} / \pi \in[0.65,0.85]$ that all four of the $\bar{X}_{0}$ states are heavier than the $X(3872)$ over the full range of $\theta_{X}$ except in the interval $\theta_{X} / \pi \in[0.29,0.42]$, and in that range only the state $\bar{X}_{0}^{\prime I=0}$ is too light. The other scalar states tend to be much heavier, ranging from at least $3900 \mathrm{MeV}$ to well over $4200 \mathrm{MeV}$. In summary, mixing angles in the ranges

$$
\frac{\theta_{X}}{\pi} \in[0,0.29],[0.42,0.79],[0.92,1], \frac{\theta_{Z}}{\pi} \in[0.65,0.85],
$$

appear to produce no conflicts with experiment.

In order to demonstrate the full predictive power of the model, we now choose one allowed set of $\left\{\theta_{X}, \theta_{Z}\right\}$ and present the complete set of mass eigenvalues for all 12 states in the ground-state $\Sigma_{g}^{+}(1 S)$ multiplet. We fix $\theta_{Z} / \pi=0.80$, in which case [by eq. (4.5)] the $Z_{c}(3900)$ is over $97 \% s_{c \bar{c}}=1$, and the original model parameters of eq. (3.9) are determined separately as

$$
M_{0}=3988.75 \mathrm{MeV}, \kappa_{q c}=17.76 \mathrm{MeV}, V_{0}=33.10 \mathrm{MeV} .
$$

Since $\left\langle e^{-m r} / r\right\rangle \approx 3.1 \mathrm{fm}^{-1}$ for the $\Sigma_{g}^{+}(1 S)$ states using $m=m_{\pi}$ (while for $m=0.5 \mathrm{GeV}$, the value is $\approx 2.0 \mathrm{fm}^{-1}$ ), eq. (3.4) gives

$$
\tilde{V}_{0}=11.0 \mathrm{MeV} \cdot \mathrm{fm},
$$

comparable in magnitude to, but a factor 2.3 larger than, the color-singlet $N N \pi$ coupling of eq. (3.3). The numerical value obtained for $\kappa_{q c}$ in eq. (4.7) is also interesting in light of the much larger corresponding value $67 \mathrm{MeV}$ obtained from a fit to exotics in the (isospinindependent) "Type-II" model [21]. Clearly, much of the strength of the coupling $\kappa_{c q}$ in the current fit has migrated to the coupling $V_{0}$. Indeed, ref. [21] notes that a fit to $\kappa_{q c}$ from the $\Sigma_{c^{-}} \Lambda_{c}$ mass difference [36] gives $\kappa_{q c}=22 \mathrm{MeV}$, which agrees much better with the result in eq. (4.7). Additionally setting $\theta_{X} / \pi=0.49$ to fix the scalar sector, we obtain 


\begin{tabular}{|ccc|}
\hline$M_{\bar{X}_{0}}^{I=0}=4215.7$ & $M_{X_{1}}^{I=0}=\mathbf{3 8 7 1 . 7}$ & $M_{\bar{Z}}^{I=0}=4271.5$ \\
$M_{\bar{X}_{0}^{\prime}}^{I=0}=3924.9$ & $M_{X_{1}}^{I=1}=4004.1$ & $M_{\bar{Z}^{\prime}}^{I=0}=3904.7$ \\
$M_{\bar{X}_{0}}^{I=1}=3936.7$ & $M_{X_{2}}^{I=0}=3907.2$ & $M_{\bar{Z}}^{I=1}=\mathbf{3 8 8 7 . 2}$ \\
$M_{\bar{X}_{0}^{\prime}}^{I=1}=3939.1$ & $M_{X_{2}}^{I=1}=4039.6$ & $M_{\bar{Z}^{\prime}}^{I=1}=\mathbf{4 0 2 4 . 1}$ \\
\hline
\end{tabular}

Table 2. Masses (in $\mathrm{MeV}$ ) of the 12 ground-state multiplet $\left[\Sigma_{g}^{+}(1 S)\right]$ states in the dynamical diquark model for the choice of mixing parameters $\theta_{X}=0.49 \pi$ and $\theta_{Z}=0.80 \pi$. Experimental inputs [eqs. (4.1)] are in boldface.

the full results presented in table 2. If $M_{0}$ in eq. (4.7) is used instead of $m_{X(3872)}$ for the $\Sigma_{g}^{+}(1 S)$ mass eigenvalue in a fit such as in ref. [18] or the right-hand columns of table 1, one obtains a diquark mass $m_{\delta}=1.92-1.94 \mathrm{GeV}$, about $3 \%$ larger, while the length scales $\langle 1 / r\rangle^{-1}$ or $\langle r\rangle$ are about $1.5 \%$ smaller.

As promised, the lowest state in this multiplet is the $X(3872)$. Its "charged partner" $X_{1}^{I=1}$ lies a full $130 \mathrm{MeV}$ higher in mass, and therefore would be expected to be quite wide, possibly unobservably so. The price for achieving this gap was noted in eq. (4.4), that the $X_{2}^{I=0}$ mass must be pushed lower, in our example to $3907.2 \mathrm{MeV}$. In fact, the $\chi_{c 2}(3930)$ has the same quantum numbers, and while expected to be the conventional charmonium $\chi_{c 2}(2 P)$ state, its most recent mass measurement by LHCb [25] of $3921.9 \pm 0.6 \pm 0.2 \mathrm{MeV}$ is rather lower than earlier determinations [35], possibly pointing to a more complicated configuration such as two peaks, or a mixture of $\chi_{c 2}(2 P)$ with a tetraquark state. The possible quasi-degeneracy of $\bar{Z}_{I=0}^{\prime}$ with $Z_{c}(3900)$ has been noted above. The $2^{++}$state $X_{2}^{I=1}$ lies near the unconfirmed $C=+$ state $Z_{c}(4055)^{ \pm}$, as well as the unconfirmed charged [40] and neutral [41] "charmoniumlike structures" around $4035 \mathrm{MeV}$.

In this particular fit, the scalar mixing angle $\theta_{X}$ was chosen to make the $0^{++}$state $\bar{X}_{0}^{\prime I=0}$ light, so as to identify it with the $\chi_{c 0}(3915)$. The nature of this state remains quite controversial [42]; for instance, it might even be the lowest $c \bar{c} s \bar{s}$ state [43]. Indeed, a very recent determination of the mass of this state as an $\omega J / \psi$ resonance [44] gives $3926.4 \pm 2.2 \mathrm{MeV}$. Meanwhile, the states $\bar{X}_{0}^{(\prime) I=1}$ are quasi-degenerate, appearing near the unconfirmed state $X(3940)$. The candidate states above $4200 \mathrm{MeV}$ are very possibly too wide to resolve experimentally. Other choices of $\theta_{X}$ can push up all of the scalar states to at least $3950 \mathrm{MeV}$, or seek to accommodate the $Z_{c}^{ \pm}(4100)$ or $X(4160)$, neither of which has been confirmed, let alone confirmed to have positive parity. The only other positive-parity states in this range, $Y(4140)$ and $Y(4274)$, are ignored in this analysis since they have only been observed as $\phi J / \psi$ resonances and therefore are very possibly $c \bar{c} s \bar{s}[18,43]$.

The dominant decay modes for exotics of these $J^{\mathrm{PC}}$ quantum numbers [37] are expected to be the $S$-wave open-charm combinations $D \bar{D}$ and $D^{*} \bar{D}^{*}$ for $0^{++}, D \bar{D}^{*}$ for $1^{++}, D \bar{D}^{*}$ and $D^{*} \bar{D}^{*}$ for $1^{+-}$, and $D^{*} \bar{D}^{*}$ for $2^{++}$(plus charge-conjugate modes, and for both $I=0$ and $I=1)$. Since $2 m_{D} \approx 3860 \mathrm{MeV}, m_{D}+m_{D^{*}} \approx 3875 \mathrm{MeV}$, and $2 m_{D^{*}} \approx 4020 \mathrm{MeV}$, most of these channels are open for the corresponding states in table 2. The dominant closedcharm modes are easily determined from eqs. (3.6) and (3.7) plus $G$-parity conservation. 
For example, $X_{2}^{I=0}$ lies below the $D^{*} \bar{D}^{*}$ threshold, and thus decays into $\omega J / \psi$, but the $D$-wave decay into $D \bar{D}$ is also possible, just as for the $\chi_{c 2}(3930)$.

Lastly, we note from table 2 that the full fine-structure splitting of the $\Sigma_{g}^{+}(1 S)$ multiplet can be much larger than the crude estimate of $150 \mathrm{MeV}$ given in ref. [18]. However, if the states heavier than $4200 \mathrm{MeV}$ turn out to be unobservably wide, then the spectrum of observable $\Sigma_{g}^{+}(1 S)$ states does indeed turn out to be about $170 \mathrm{MeV}$.

\section{Discussion and conclusions}

In this paper we have developed a variant of the dynamical diquark model in which isospin dependence is explicitly incorporated. We also developed a simple modification of our calculation to test for effects on the exotics spectrum due to finite diquark size.

Allowing for the potential to transition from one describing the interaction of diquarks at large separations $r$ to one dominated by the $Q \bar{Q}$ interaction at separations below a chosen value $r=R$ effectively introduces an effective diquark radius of $R / 2$. We find by explicit calculation that the exotics spectrum changes very little until $R$ is as large as $0.8 \mathrm{fm}$, meaning that results obtained by treating the diquarks as pointlike are reliable even for compact diquarks with radii as large as $0.4 \mathrm{fm}$.

The existence of an isospin-dependent interaction between separated diquarks, a type of (partly) colored pion exchange, is inspired by the existence of a Nambu-Goldstone theorem of chiral-symmetry breaking shown to occur in dense QCD. Isospin dependence is clearly evident in the observation of exotic states to appear in isosinglets and isotriplets, rather than quartets. We applied this ansatz of isospin dependence to the 12 states in the groundstate multiplet $\Sigma_{g}^{+}(1 S)$, taking $X(3872), Z_{c}(3900)$, and $Z_{c}(4020)$ as members, and predicted the masses of the others.

The $X(3872)$ in this model naturally emerges over large portions of the allowed parameter space as the lightest exotic state, and its "charged partner", the $J^{\mathrm{PC}}=1^{++} I=1$ member of the multiplet, is much heavier. Moreover, the decay preferences $Z_{c}(3900) \rightarrow J / \psi$ and $Z_{c}(4020) \rightarrow h_{c}$ emerge directly from the analysis of the mass spectrum. We have obtained fits in which several of the ill-characterized low-lying exotics naturally appear as members of the $\Sigma_{g}^{+}(1 S)$ multiplet, and some of the predicted mass eigenvalues lie so high above the dominant "fall-apart" decay mode of the corresponding state that they may be too wide to discern easily.

The natural next step is to consider the first excited multiplet, $\Sigma_{g}^{+}(1 P)$, whose states all carry negative parity. A number of states have been assigned to this multiplet [18], such as $Y(4220)$ and $Y(4360)$. However, both experimental and theoretical issues complicate this analysis. This mass region includes the expected location of the lightest hybrid charmonium states [17], which lie outside this analysis. Additionally, no $P=-, I=1$ exotic states have yet been confirmed. From the perspective of modeling, several other operators not included in eq. (3.9) need to be considered, not least of which are the tensor operator $S_{12}$ of eq. (3.2) (both isospin-dependent and independent) and the spin-orbit operator. In the second excited multiplet [including states such as $Z_{c}(4430)$ ], one expects the range of masses of states in either $\Sigma_{g}^{+}(2 S)$ or $\Sigma_{g}^{+}(1 D)$ to overlap [18], or even for the states 
themselves in the two multiplets to mix via tensor terms, again complicating the analysis. In short, not enough states have been fully characterized in the excited multiplets of this model to perform a reliable analysis.

Nevertheless, one basic feature is expected to hold for the excited multiplets: with reference to eq. (3.4), excited states are spatially larger, meaning that $\left\langle e^{-m_{\pi} r} / r\right\rangle$ is smaller in higher levels, and so one expects smaller mass splittings within the higher multiplets in this model (an analogous effect occurs for fine-structure splittings in ordinary quarkonium).

Multiplets of exotics with excited glue fields, such as $\Pi_{u}^{+}(1 P)-\Sigma_{u}^{-}(1 P)$, have not even been mentioned in this paper, since as was shown in ref. [18], they are expected to lie about $1 \mathrm{GeV}$ above the $\Sigma_{g}^{+}(1 S)$ ground states (just like the gap between quarkonium hybrids and conventional quarkonium states). Nevertheless, were they to be considered in a model analogous to the one described here, yet further operators would need to be included, such as ones dependent upon not only quark spin, but the spin of the nontrivial glue degrees of freedom as well [45].

Lastly, all of the phenomenology presented here refers to the sector of hidden-charm tetraquarks. In the pentaquark sector, the states according to the model of ref. [13] contain triquarks of the form $\bar{\theta}=\left[\bar{c}(u d)_{\overline{\mathbf{3}}}\right]_{\mathbf{3}}$, where the $u d$ pair is an $I=0$ diquark inherited from the initial $\Lambda_{b}$ decay process from which all pentaquarks to date have been produced. But in that case, the $\bar{\theta}-\delta$ pair does not exchange isospin, and only simple $I=\frac{1}{2}$ pentaquarks occur. Likewise, if the $u d$ diquark carries spin 0 like that in $\Lambda_{b}$, then the triquark uniquely carries spin $\frac{1}{2}$. Transitions to the higher-mass "bad" $(I=1$, spin-1) light diquark are certainly possible, but are expected to be suppressed. One may then study the pentaquarks in a spin-only formulation of the model, as in, e.g., ref. [46], or using a different diquark-triquark formulation as in ref. [47].

Nor has the $b$ sector been discussed in this paper. Again, not enough states have been observed to attempt a reliable fit to the full spectrum, but in this case the bestcharacterized exotic candidates are isotriplets, the $Z_{b}(10610)$ and $Z_{b}(10650)$. The relative spacings of $B^{(*)} \bar{B}^{(*)}$ thresholds and conventional bottomonium levels are different from those in the $c \bar{c}$ system, leading to a rather different phenomenology. The bottom analogue to the $X(3872)$, the $I=01^{++}$state $X_{b}$ (see refs. [48,49] for nice discussions of its expected properties) has not yet been observed. While $X(3872)$ emerged naturally as the lightest state among the hidden-charm tetraquarks in this model, there exist alternate portions of the parameter space where the $I=01^{++}$state is not the lightest, and this observation may turn out to be relevant for the $b$ system.

\section{Acknowledgments}

R.F.L. and J.F.G. were supported by the National Science Foundation (NSF) under Grant No. PHY-1803912; J.F.G. also received support through the Western Alliance to Expand Student Opportunities (WAESO) Louis Stokes Alliance for Minority Participation Bridge to the Doctorate (LSAMPBD) NSF Cooperative Agreement HRD-1702083; and C.T.P. through a NASA traineeship grant awarded to the Arizona/NASA Space Grant Consortium. 
Open Access. This article is distributed under the terms of the Creative Commons Attribution License (CC-BY 4.0), which permits any use, distribution and reproduction in any medium, provided the original author(s) and source are credited.

\section{References}

[1] R.F. Lebed, R.E. Mitchell and E.S. Swanson, Heavy-quark QCD exotica, Prog. Part. Nucl. Phys. 93 (2017) 143 [arXiv:1610.04528] [INSPIRE].

[2] H.-X. Chen, W. Chen, X. Liu and S.-L. Zhu, The hidden-charm pentaquark and tetraquark states, Phys. Rept. 639 (2016) 1 [arXiv:1601.02092] [InSPIRE].

[3] A. Hosaka et al., Exotic hadrons with heavy flavors: X, Y, Z and related states, PTEP 2016 (2016) $062 \mathrm{C} 01$ [arXiv: 1603.09229] [INSPIRE].

[4] A. Esposito, A. Pilloni and A.D. Polosa, Multiquark resonances, Phys. Rept. 668 (2017) 1 [arXiv: 1611.07920] [INSPIRE].

[5] F.-K. Guo et al., Hadronic molecules, Rev. Mod. Phys. 90 (2018) 015004 [arXiv: 1705.00141] [INSPIRE].

[6] A. Ali, J.S. Lange and S. Stone, Exotics: heavy pentaquarks and tetraquarks, Prog. Part. Nucl. Phys. 97 (2017) 123 [arXiv:1706.00610] [INSPIRE].

[7] S.L. Olsen, T. Skwarnicki and D. Zieminska, Nonstandard heavy mesons and baryons: experimental evidence, Rev. Mod. Phys. 90 (2018) 015003 [arXiv:1708.04012] [InSPIRE].

[8] M. Karliner, J.L. Rosner and T. Skwarnicki, Multiquark states, Ann. Rev. Nucl. Part. Sci. 68 (2018) 17 [arXiv:1711.10626] [INSPIRE].

[9] C.-Z. Yuan, The XYZ states revisited, Int. J. Mod. Phys. A 33 (2018) 1830018 [arXiv: 1808.01570] [INSPIRE].

[10] Y.-R. Liu et al., Pentaquark and tetraquark states, Prog. Part. Nucl. Phys. 107 (2019) 237 [arXiv: 1903.11976] [INSPIRE].

[11] N. Brambilla et al., The XYZ states: experimental and theoretical status and perspectives, arXiv: 1907.07583 [INSPIRE].

[12] S.J. Brodsky, D.S. Hwang and R.F. Lebed, Dynamical picture for the formation and decay of the exotic XYZ mesons, Phys. Rev. Lett. 113 (2014) 112001 [arXiv:1406.7281] [inSPIRE].

[13] R.F. Lebed, The pentaquark candidates in the dynamical diquark picture, Phys. Lett. B 749 (2015) 454 [arXiv:1507.05867] [INSPIRE].

[14] S.J. Brodsky and R.F. Lebed, QCD dynamics of tetraquark production, Phys. Rev. D 91 (2015) 114025 [arXiv: 1505.00803] [INSPIRE].

[15] R.F. Lebed, Spectroscopy of exotic hadrons formed from dynamical diquarks, Phys. Rev. D 96 (2017) 116003 [arXiv: 1709.06097] [INSPIRE].

[16] M. Born and R. Oppenheimer, Zür Quantentheorie der Molekeln, Ann. Phys. 389 (1927) 457.

[17] HADRon SPECTRUm collaboration, Excited and exotic charmonium spectroscopy from lattice QCD, JHEP 07 (2012) 126 [arXiv: 1204.5425] [INSPIRE].

[18] J.F. Giron, R.F. Lebed and C.T. Peterson, The dynamical diquark model: first numerical results, JHEP 05 (2019) 061 [arXiv: 1903.04551] [INSPIRE]. 
[19] K.J. Juge, J. Kuti and C. Morningstar, Fine structure of the QCD string spectrum, Phys. Rev. Lett. 90 (2003) 161601 [hep-lat/0207004] [INSPIRE].

[20] S. Capitani et al., Precision computation of hybrid static potentials in $\mathrm{SU}(3)$ lattice gauge theory, Phys. Rev. D 99 (2019) 034502 [arXiv:1811.11046] [INSPIRE].

[21] L. Maiani, F. Piccinini, A.D. Polosa and V. Riquer, The $Z(4430)$ and a new paradigm for spin interactions in tetraquarks, Phys. Rev. D 89 (2014) 114010 [arXiv:1405.1551] [INSPIRE].

[22] S.K. Choi et al., Bounds on the width, mass difference and other properties of $X(3872) \rightarrow \pi^{+} \pi^{-} J / \psi$ decays, Phys. Rev. D 84 (2011) 052004 [arXiv:1107.0163] [inSPIRE].

[23] L. Ya. Glozman and D.O. Riska, The spectrum of the nucleons and the strange hyperons and chiral dynamics, Phys. Rept. 268 (1996) 263 [hep-ph/9505422] [INSPIRE].

[24] M.G. Alford, K. Rajagopal and F. Wilczek, Color flavor locking and chiral symmetry breaking in high density QCD, Nucl. Phys. B 537 (1999) 443 [hep-ph/9804403] [InSPIRE].

[25] LHCb collaboration, Near-threshold D $\bar{D}$ spectroscopy and observation of a new charmonium state, JHEP 07 (2019) 035 [arXiv: 1903.12240] [INSPIRE].

[26] Belle collaboration, Observation of an alternative $\chi_{c 0}(2 P)$ candidate in $e^{+} e^{-} \rightarrow J / \psi D \bar{D}$, Phys. Rev. D 95 (2017) 112003 [arXiv:1704.01872] [INSPIRE].

[27] M.B. Voloshin, Deciphering the XYZ states, in the proceedings of the $17^{\text {th }}$ Conference on Flavor Physics and CP-violation (FPCP 2019), May 6-10, Victoria, Canada (2019), arXiv: 1905.13156 [INSPIRE].

[28] L. Maiani, A.D. Polosa and V. Riquer, Hydrogen bond of QCD, Phys. Rev. D 100 (2019) 014002 [arXiv: 1903.10253] [INSPIRE].

[29] S. Dubynskiy and M.B. Voloshin, Hadro-charmonium, Phys. Lett. B 666 (2008) 344 [arXiv: 0803.2224] [INSPIRE].

[30] M. Berwein, N. Brambilla, J. Tarrús Castellà and A. Vairo, Quarkonium hybrids with nonrelativistic effective field theories, Phys. Rev. D 92 (2015) 114019 [arXiv:1510.04299] [INSPIRE].

[31] http://www.andrew.cmu.edu/user/cmorning/static_potentials/SU3_4D/greet.html

[32] N. Kemmer, Nature of the nuclear field, Nature 141 (1938) 116.

[33] N. Kemmer, Quantum theory of Einstein-Bose particles and nuclear interaction, Proc. Roy. Soc. A 166 (1938) 127.

[34] M. Taketani, S. Nakamura and M. Sasaki, On the method of the theory of nuclear forces, Prog. Theor. Phys. 6 (1951) 581.

[35] Particle Data Group collaboration, Review of particle physics, Phys. Rev. D 98 (2018) 030001 [INSPIRE].

[36] L. Maiani, F. Piccinini, A.D. Polosa and V. Riquer, Diquark-antidiquarks with hidden or open charm and the nature of X(3872), Phys. Rev. D 71 (2005) 014028 [hep-ph/0412098] [INSPIRE].

[37] M. Cleven et al., Employing spin symmetry to disentangle different models for the XYZ states, Phys. Rev. D 92 (2015) 014005 [arXiv: 1505.01771] [INSPIRE]. 
[38] BESIII collaboration, Observation of the decay $X(3872) \rightarrow \pi^{0} \chi_{c 1}(1 P)$, Phys. Rev. Lett. 122 (2019) 202001 [arXiv: 1901.03992] [INSPIRE].

[39] BESIII collaboration, Study of $e^{+} e^{-} \rightarrow \pi^{+} \pi^{-} \pi^{0} \eta_{c}$ and evidence for $Z_{c}(3900)^{ \pm}$decaying into $\rho^{ \pm} \eta_{c}$, Phys. Rev. D 100 (2019) 111102 [arXiv:1906.00831] [INSPIRE].

[40] BESIII collaboration, Measurement of $e^{+} e^{-} \rightarrow \pi^{+} \pi^{-} \psi(3686)$ from 4.008 to $4.600 \mathrm{GeV}$ and observation of a charged structure in the $\pi^{ \pm} \psi(3686)$ mass spectrum, Phys. Rev. D 96 (2017) 032004 [arXiv: 1703.08787] [INSPIRE].

[41] BESIII collaboration, Measurement of $e^{+} e^{-} \rightarrow \pi^{0} \pi^{0} \psi(3686)$ at $\sqrt{s}$ from 4.009 to $4.600 \mathrm{GeV}$ and observation of a neutral charmoniumlike structure, Phys. Rev. D 97 (2018) 052001 [arXiv: 1710.10740] [INSPIRE].

[42] S.L. Olsen, Comment on the X(3915) nonstandard hadron candidate, EPJ Web Conf. 212 (2019) 02009 [arXiv:1904.06130] [INSPIRE].

[43] R.F. Lebed and A.D. Polosa, $\chi_{c 0}(3915)$ as the lightest $c \bar{c} s \bar{s}$ state, Phys. Rev. D 93 (2016) 094024 [arXiv: 1602.08421] [INSPIRE].

[44] BESIII collaboration, Study of $e^{+} e^{-} \rightarrow \gamma \omega J / \psi$ and Observation of $X(3872) \rightarrow \omega J / \psi$, Phys. Rev. Lett. 122 (2019) 232002 [arXiv:1903.04695] [INSPIRE].

[45] N. Brambilla et al., Spin structure of heavy-quark hybrids, Phys. Rev. D 99 (2019) 014017 [arXiv: 1805.07713] [INSPIRE].

[46] R. Zhu and C.-F. Qiao, Pentaquark states in a diquark-triquark model, Phys. Lett. B 756 (2016) 259 [arXiv: 1510.08693] [InSPIRE].

[47] A. Ali et al., Mass spectrum of the hidden-charm pentaquarks in the compact diquark model, JHEP 10 (2019) 256 [arXiv: 1907.06507] [INSPIRE].

[48] F.-K. Guo, U.-G. Meissner, W. Wang and Z. Yang, Production of the bottom analogs and the spin partner of the X(3872) at hadron colliders, Eur. Phys. J. C 74 (2014) 3063 [arXiv: 1402.6236] [INSPIRE].

[49] M. Karliner and J.L. Rosner, $X(3872), X_{b}$ and the $\chi_{b 1}(3 P)$ state, Phys. Rev. D 91 (2015) 014014 [arXiv: 1410.7729] [INSPIRE]. 